\title{
Gold nanoparticles promote osteogenic differentiation in human adipose-derived mesenchymal stem cells through the $\mathrm{Wnt} / \mathrm{\beta}$ - catenin signaling pathway
}

\author{
This article was published in the following Dove Press journal: \\ International Journal of Nanomedicine \\ 7 July 2015 \\ Number of times this article has been viewed
}

\section{Seon Young Choi \\ Min Seok Song' \\ Pan Dong Ryu' \\ Anh Thu Ngoc Lam² \\ Sang-Woo Joo ${ }^{2}$ \\ So Yeong Lee'}

'Laboratory of Veterinary

Pharmacology, Research Institute for Veterinary Science, College of Veterinary Medicine, Seoul National University, ${ }^{2}$ Department of Chemistry, Soongsil University, Seoul, South Korea

Correspondence: So Yeong Lee Laboratory of Veterinary Pharmacology, Research Institute for Veterinary Science, College of Veterinary Medicine, Seoul National University, I Gwanak-ro Gwanak-gu, Seoul I5I-742, South Korea $\mathrm{Tel}+822880 \mathrm{I} 283$

Fax +82 28790378

Email leeso@snu.ac.kr

Sang-Woo Joo

Department of Chemistry, Soongsil

University, 369 Sangdo-Ro, Dongjak-gu,

Seoul I56-743, South Korea

Tel +8228200434

Fax +82 28200434

Email sjoo@ssu.ac.kr
Abstract: Gold nanoparticles (AuNPs) are attractive materials for use in biomedicine due to their physical properties. Increasing evidence suggests that several nanoparticles induce the differentiation of human mesenchymal stem cells into osteoblasts and adipocytes. In this study, we hypothesized that chitosan-conjugated AuNPs promote the osteogenic differentiation of human adipose-derived mesenchymal stem cells. For the evaluation of osteogenic differentiation, alizarin red staining, an alamarBlue ${ }^{\circledR}$ assay, and a quantitative real-time polymerase chain reaction analysis were performed. In order to examine specific signaling pathways, immunofluorescence and a western blotting assay were performed. Our results demonstrate that chitosan-conjugated AuNPs increase the deposition of calcium content and the expression of marker genes related to osteogenic differentiation in human adipose-derived mesenchymal stem cells at nontoxic concentrations. These results indicate that chitosan-conjugated AuNPs promote osteogenesis through the Wnt/ $\beta$-catenin signaling pathway. Therefore, chitosan-conjugated AuNPs can be used as a reagent for promoting bone formation.

Keywords: chitosan-conjugated gold nanoparticle, mineralization, nonphosphorylated betacatenin

\section{Introduction}

Transplant engineering of stem cells is a potential therapeutic method for the regeneration of damaged tissue, such as with osteoporosis, which is becoming an important issue and health problem. ${ }^{1-6}$ Human mesenchymal stem cells (hMSCs) derived from various tissues, including bone marrow, adipose tissue, and umbilical cord blood, possess multilineage differentiating potentials. ${ }^{7}$ Interests in the therapeutic potential of stem cells isolated from adipose tissue, called adipose-derived stem cells (ADSCs), has grown due to a less expensive acquisition and greater available quantities than bone marrow. ${ }^{8-11}$ However, mesenchymal stem cells (MSCs) derived from various sources have limited proliferation activity and differentiation potentials. ${ }^{12,13}$ Therefore, it appears that developing new technologies to stimulate the differentiation of MSCs is required. Recently, gold nanoparticles (AuNPs) have been suggested as useful medical vehicles for regenerative tools due to their nontoxic effects, as compared to other types of nanoparticles (NPs). ${ }^{14,15}$ Various forms of AuNPs are known to be involved in the differentiation of hMSCs into various types of cells. For example, gold-coated iron oxide ( $\mathrm{gold} / \mathrm{Fe}_{3} \mathrm{O}_{4}$ ) and graphene stimulate the neural differentiation of rat olfactory bulb neural stem cells, and graphene oxide-encapsulated AuNPs are attractive 
detection materials for neural stem cell differentiation. ${ }^{16,17}$ In addition, it has been proposed that AuNPs regulate the myocardiac differentiation of MSCs. ${ }^{18,19}$ Many reports suggest that AuNPs promote the differentiation of hMSCs into osteoblasts. ${ }^{20,21}$ Citrate-reduced AuNPs stimulate the osteogenic differentiation of bone marrow-derived MSCs through the mitogen-activated protein kinase signaling pathway, and the osteogenic differentiation of fibroblasts was enhanced by an AuNPs-hydrogel complex through the bone morphogenic protein signaling pathway. ${ }^{22,23}$ Influence of various nanomaterials on stem cell differentiation was studied; ${ }^{16-24}$ however, research regarding the effects of chitosan-conjugated AuNPs on the differentiation of hMSCs has not been reported.

In the present study, we investigated the effect of chitosan-conjugated AuNPs on the differentiation of human adipose-derived mesenchymal stem cells (hADMSCs) as a switch to determine cell fate into osteoblasts and the relating signaling pathway in cell differentiation.

\section{Materials and methods Culture of hADMSCs}

The hADMSCs were purchased from ATCC (PCS-500-011) and cultured in low-glucose Dulbecco's Modified Eagle's Medium (GIBCO cell culture, Thermo Fisher Scientific, Waltham, MA, USA) with 10\% fetal bovine serum and 1\% antibiotics (Sigma-Aldrich, St Louis, MO, USA) incubating at $37^{\circ} \mathrm{C}$ in $5 \% \mathrm{CO}_{2}$. The culture medium was replaced every 3 days during the experiments.

\section{Characterization of chitosan-conjugated AuNPs}

In this study, we used the same chitosan-conjugated AuNPs which were used in our previous study. ${ }^{25}$ In summary, positively charged AuNPs were prepared using the chitosan reduction method. The average diameter of chitosan-conjugated AuNPs was $\sim 17 \mathrm{~nm}$, and the zeta potentials were $\sim 42 \mathrm{mV}$. The hydrodynamic diameters of chitosan-conjugated AuNPs were $\sim 40 \mathrm{~nm}$ from the dynamic light scattering measurements.

\section{Transmission electron microscope}

The uptake of chitosan-conjugated AuNPs was examined using a transmission electron microscope (TEM). The hADMSCs were plated into six-well culture plates at $3 \times 103$ cells $/ \mathrm{cm}^{2}$. After cell confluency, chitosan-conjugated AuNPs were added to the growth medium and incubated at $37^{\circ} \mathrm{C}$ in $5 \% \mathrm{CO}_{2}$ for 24 hours. Fixation, embedding, ultrathin sections, and analysis using a TEM were operated in the Integrative Research Support Center in School of Medicine, the Catholic University of Korea.

\section{Cell viability assay}

The cell viability of hADMSCs was assessed using a Cell Counting Kit-8 (CCK-8; Dojindo Molecular Technologies, Inc. Kumamoto, Japan). Cells were seeded in 96-well culture plates at $3 \times 10^{3}$ cells $/ \mathrm{cm}^{2}$. After cell confluency, chitosanconjugated AuNPs were added to the culture medium. Wells containing the culture medium and NPs without cells were used as blanks. After 72 hours of incubation, $10 \mu \mathrm{L}$ of the CCK-8 solution was added to the medium and incubated for 4 hours at $37^{\circ} \mathrm{C}$. The absorbance of the optical density (OD) at $450 \mathrm{~nm}$ was measured using a microplate reader (TECAN, Männedorf, Switzerland). The cell viability (\%) was calculated according to the formula: $\left(\mathrm{OD}_{\text {sample }}-\mathrm{OD}_{\text {blank }}\right) /\left(\mathrm{OD}_{\text {control }}-\mathrm{OD}_{\text {blank }}\right) \times 100$.

\section{Proliferation of hADMSCs}

The proliferation of the hADMSCs was determined using an alamarBlue $^{\circledR}$ assay. Briefly, hADMSCs were seeded in each well of 96-well plates at a density of $3 \times 10^{3}$ cells $/ \mathrm{cm}^{2}$ and incubated for 72 hours. After cell confluency, an osteogenicinducing medium (OM) containing $100 \mathrm{nM}$ dexamethasone (Calbiochem, EMD Millipore, Billerica, MA, USA), $50 \mu \mathrm{M}$ L-ascorbic acid 2-phosphate sesquimagnesium salt hydrate (Sigma-Aldrich), and $10 \mathrm{mM}$ beta-glycerophosphate disodium salt hydrate (Sigma-Aldrich) was added to each well with different concentrations of chitosan-conjugated AuNPs and incubated for 10, 14, and 21 days. Wells containing OM and NPs without cells were used as blanks. At each time point, $20 \mu \mathrm{L}$ alamarBlue ${ }^{\circledR}$ solution (Invitrogen Corporation, Carlsbad, CA, USA) was added to the medium and incubated for 4 hours at $37^{\circ} \mathrm{C}$. The absorbances at $570 \mathrm{~nm}$ and $620 \mathrm{~nm}$ were measured using a microplate reader (TECAN).

\section{Alizarin Red S for mineralization}

The osteogenic differentiation rates of hADMSCs in the presence of chitosan-conjugated AuNPs were determined by an Alizarin Red S (ARS) staining assay. The hADMSCs were seeded in a six-well culture plates at a cell density of $3 \times 10^{3}$ cells $/ \mathrm{cm}^{2}$ and cultured for 10,14 , and 21 days in OM and chitosan-conjugated AuNPs. Briefly, the cells were fixed with $80 \%$ ethanol for 1 hour at room temperature. After being washed with distilled water, they were stained with $60 \mathrm{mM}$ ARS ( $\mathrm{pH} 4.2$ ) for 15 minutes at room temperature. A quantitation of ARS staining was performed by elution with $10 \%(\mathrm{w} / \mathrm{v})$ cetylpyridinium chloride (Sigma-Aldrich) 
for 20 minutes at room temperature, and the absorbance was measured at $570 \mathrm{~nm}$. The mineralization ratio was normalized to the proliferation ratio.

\section{Oil Red $O$ for lipid accumulation}

The adipogenic differentiation rates of hADMSCs in the presence of chitosan-conjugated AuNPs were determined by an Oil Red O (ORO) staining assay. After osteogenic induction, the cells were washed with distilled water and they were stained with $0.6 \%$ (w/v) ORO solution (60\% isopropanol) for 15 minutes at room temperature. For quantitation, the cells were washed with distilled water to remove unbound dye, and $2 \mathrm{~mL}$ isopropyl alcohol was added to the wells of the culture plates. After 20 minutes, the absorbance was measured by a microplate reader at $510 \mathrm{~nm}$. The lipid accumulation ratio was normalized to the proliferation ratio.

\section{Real-time RT-PCR analysis}

The total RNA was isolated from the differentiated cells, which were treated with chitosan-conjugated AuNPs for 10, 14, and 21 days using a Hybrid-R prep kit (GeneAll Biotechnology Co., Ltd., Seoul, South Korea) according to the manufacturer's protocol. Reverse transcription (RT) was performed using M-MLV reverse transcriptase (Invitrogen). The relative mRNA expression of the osteogenic differentiation marker genes was normalized to the $G A P D H$ gene and expressed as a fold change relative to the growth medium group. The polymerase chain reaction (PCR) conditions were an initial step at $95^{\circ} \mathrm{C}$ for 30 seconds and 40 denaturation cycles of $95^{\circ} \mathrm{C}$ for 5 seconds and annealing at $60^{\circ} \mathrm{C}$ for 45 seconds. Steps at $95^{\circ} \mathrm{C}$ for 15 seconds, $60^{\circ} \mathrm{C}$ for 1 minute, and $95^{\circ} \mathrm{C}$ for 15 seconds was added to minimize nonspecific products. The results were analyzed by comparing the $2^{-[\text {delta][delta]Ct }}$ values of the mRNA of cells treated with chitosan-conjugated AuNPs to those of the cells in $\mathrm{OM}$. Table 1 represents the list of primers used for real-time RT-PCR.

\section{Western blotting analysis}

A western blotting assay was applied to study the protein expressions of hADMSCs related to the $\beta$-catenin signaling pathway. Proteins were extracted and subjected to $10 \%$ sodium dodecyl sulphate-polyacrylamide gel electrophoresis (SDS-PAGE) for the detection of active-form $\beta$-catenin, then transferred to PVDF membranes. The membranes were blocked with 5\% skim milk in tris-buffered saline with Tween 20 for 1 hour at room temperature. Membranes were reacted with primary antibodies overnight at $4^{\circ} \mathrm{C}$. Primary antibodies were as follows: anti-nonphosphorylated $\beta$-catenin (1:500 [EMD Millipore, Billerica, MA, USA]) and $\beta$-actin (1:1000 [Santa Cruz Biotechnology Inc., Dallas, TX, USA]), and the $\beta$-actin protein was used as the control.

\section{Immunofluorescence for translocation of nonphosphorylated $\beta$-catenin into the nucleus}

The hADMSCs were plated to confocal dishes (Nunc ${ }^{\text {TM }}$ LabTek chamber slides, Thermo Fisher Scientific) at a density of $3 \times 10^{3}$ cells $/ \mathrm{cm}^{2}$ and cultured for $7,10,14$, and 21 days in an OM treated with chitosan-conjugated AuNPs. The cells were fixed with $4 \%$ paraformaldehyde for 15 minutes. Fixed cells were incubated with an active-form $\beta$-catenin (EMD Millipore) antibody for 48 hours at $4^{\circ} \mathrm{C}$, then incubated with a secondary antibody, which was conjugated with Alexa Flour $^{\circledR} 555$ for 1 hour at room temperature. Cells were stained with 2-(4-amidinophenyl)-6-indolecarbamidine dihydrochloride for nucleus staining. Confocal images were acquired with a LSM 710 confocal laser scanning microscope (Carl Zeiss, Hamburg, Germany) operated in the National Center for Inter-University Research Facilities (Seoul National University, Korea).

\section{Statistical analysis}

Data was generally presented as the mean \pm standard error, and the statistical differences between the experimental

Table I Primers for quantitative real-time RT-PCR analysis

\begin{tabular}{llr}
\hline Gene (accession number) & Sequences & Product size (bp) \\
\hline Alkaline phosphatase (NM_000478.4) & F 5'-CCTCCTCGGAAGACACTCTG-3' & I39 \\
Bone sialoprotein (NM_004967.3) & R 5'-GCAGTGAAGGGCTTCTTGTC-3' & $16 \mathrm{I}$ \\
Osteocalcin (NM_00I 199662.1) & F 5'-AAAGTGAGAACGGGGAACCT-3' & 119 \\
GAPDH (NM_002046.4) & R 5'-GATGCAAAGCCAGAATGGAT-3' & 112 \\
& F 5'-GACTGTGACGAGTTGGCTGA-3' & R 5'-CTGGAGAGGAGCAGAACTGG-3' \\
\hline
\end{tabular}

Abbreviations: $F$, forward; $R$, reverse; RT-PCR, reverse transcription polymerase chain reaction. 
groups were analyzed by Student's $t$-test using statistical software Origin 8.0. $P<0.05$ was considered statistically significant in all cases.

\section{Results and discussion Uptake of chitosan-conjugated AuNPs in hADMSCs}

Recent studies suggested that the anionic AuNPs are endocytosed into cells, while half the amounts of cationic AuNPs escape the endocytotic pathway. ${ }^{26}$ Cationic AuNPs may directly diffuse into cytosol by generating disruptions on the cell membranes..$^{27,28}$ In addition, uptake mechanisms of NPs were reportedly dependent on the cell types. ${ }^{29,30}$ In an earlier study, chitosan-conjugated AuNPs demonstrated endocytotic uptake in lung cancer cells. ${ }^{25}$ In order to identify uptake of chitosan-conjugated AuNPs in hADMSCs, internalized chitosan-conjugated AuNPs were measured using a TEM. The concentration of chitosan-conjugated AuNPs added for TEM was $1 \mathrm{ppm}$. Most of the internalized chitosan-conjugated AuNPs were detected in cytosol as shown in Figure 1. However, in this TEM images, chitosan-conjugated AuNPs internalized into hADMSCs were not inside endosomal vesicles. According to our results, chitosan-conjugated AuNPs entered into hADMSCs through direct diffusion or escaping the endocytotic pathway.

\section{Effect of chitosan-conjugated AuNPs on hADMSC viability and proliferation}

Over the past few years, the interactions of AuNPs with cell have been researched to confirm their uptake, distribution, and cytotoxicity. AuNPs with various sizes and shapes were taken in a variety of cell types. Recent evidence indicated that AuNPs decreased cell viability and differentiation of bone marrow MSCs through triggered necrosis by excessive reactive oxygen species (ROS) generation. ${ }^{31}$ In particular, excessive ROS cause damage to MSCs, whereas low levels of ROS enhance osteogenesis of MSCs. ${ }^{32}$ To determine the viability of hADMSCs treated with chitosan-conjugated AuNPs, cell viability assays were performed for 72 hours in a growth medium that did not contain osteogenic-inducing substances (Figure 2A). As shown in Figure 2A, chitosan-conjugated AuNPs had no effect on the cell viability of hADMSCs at a concentration of up to $10 \mathrm{ppm}$. The enhancement of the osteogenic differentiation was followed by increased cell proliferation. ${ }^{7,33}$ To determine the proliferation of the hADMSCs in osteogenic-inducing conditions, a proliferation assay was examined at 10,14, and 21 days in an OM-containing chitosan-conjugated AuNPs (Figure 2B). This result indicates that the value of OD was significantly increased in all experimental groups in a time-dependent manner. However, there is no statistical significance in the difference between the OM-only group and the OM-containing chitosan-conjugated AuNPs group. The degree of uptake and subcellular localization in cell systems showed different cytotoxicity. ${ }^{34}$ In particular, gold nanostars located in the nucleus are more toxic compared to the gold nanostars located in the cytosol. ${ }^{35}$ Therefore, it is possible that AuNPs located in membrane are lacking in cytotoxicity in hADMSCs.

\section{Chitosan-conjugated AuNPs promote the osteogenic differentiation of hADMSCs}

Pristine AuNPs without any appropriate modifications are generally unstable because of high surface energy. So a stabilizer should be added to apply AuNPs into cells. ${ }^{36}$ In the present study, chitosan was used in the reduction of AuNPs as a stabilizer. Several researches suggest that
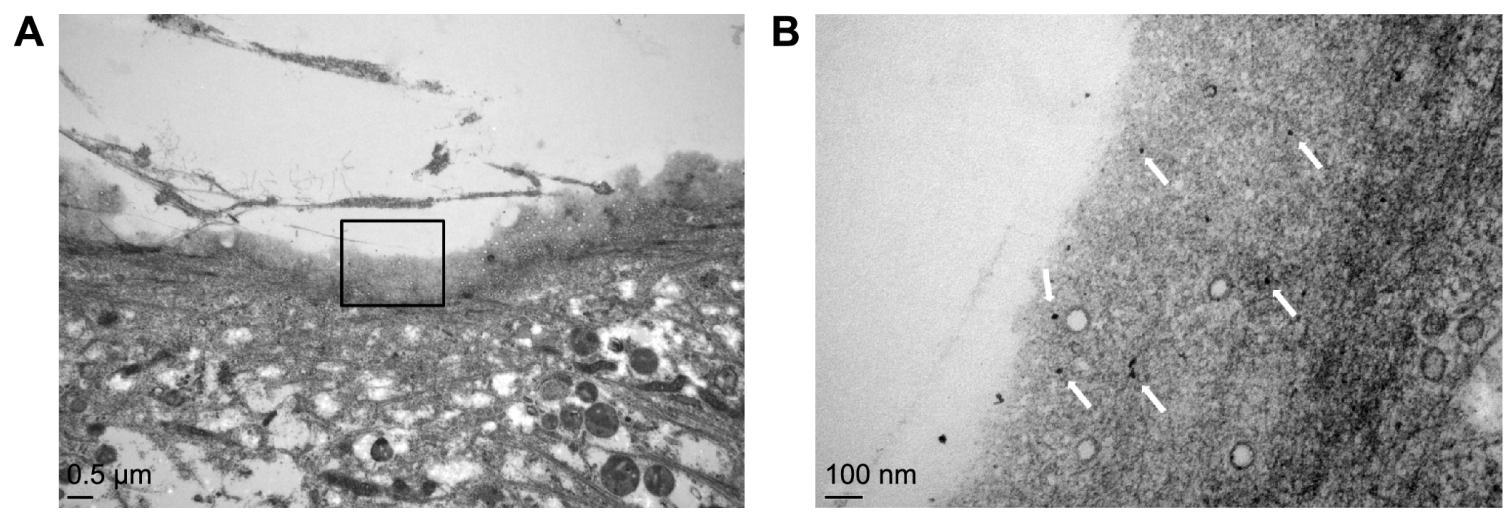

Figure I TEM images of hADMSCs exposed to chitosan-conjugated AuNPs.

Notes: hADMSCs were exposed to chitosan-conjugated AuNPs for 24 hours and then fixed for TEM. (A) Chitosan-conjugated AuNPs were internalized in cell membrane as magnified in the (B) image. White arrows represent chitosan-conjugated AuNPs in cell membrane. The scale bars in (A) and (B) are $0.5 \mu \mathrm{m}$ and I00 $\mathrm{nm}$.

Abbreviations: AuNPs, gold nanoparticles; hADMSCs, human adipose-derived mesenchymal stem cells; TEM, transmission electron microscope. 

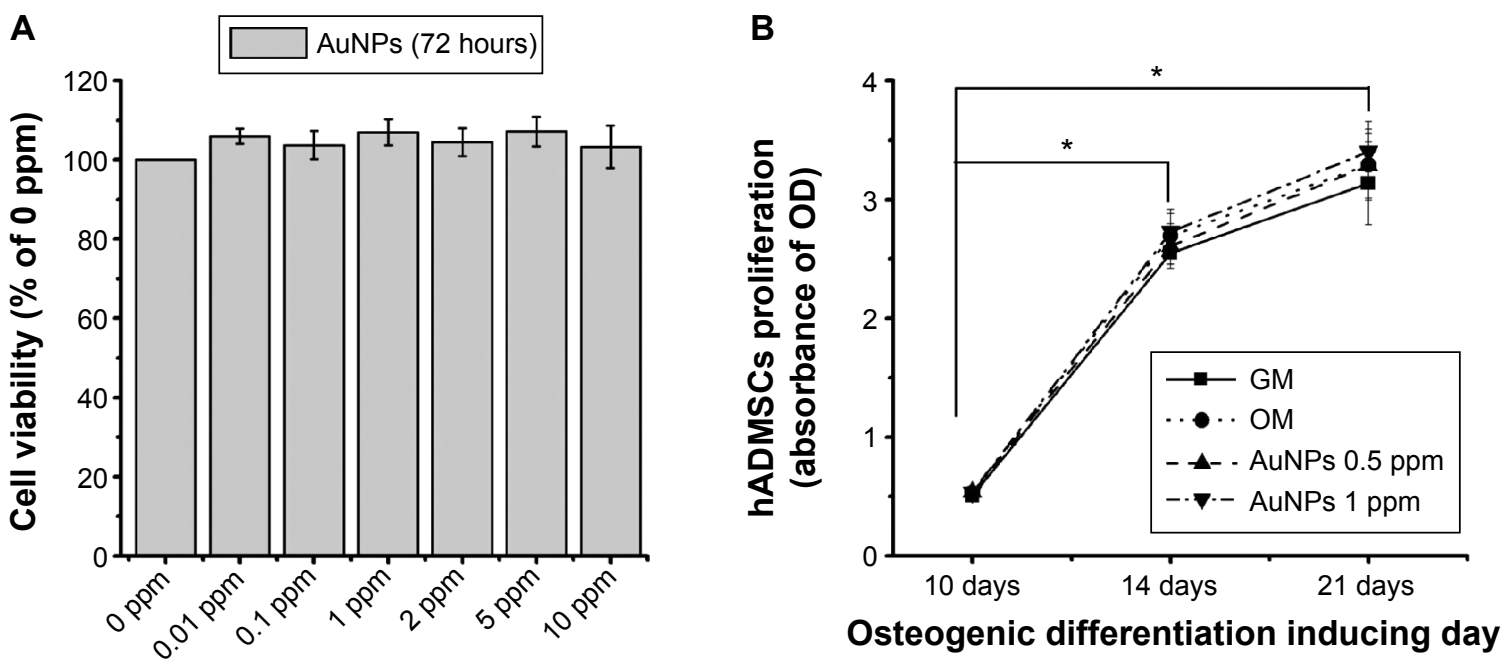

Figure 2 Effects of chitosan-conjugated AuNPs.

Notes: (A) On the cell viability of hADMSCs for 72 hours in a growth medium (GM) and (B) on the cell proliferation of hADMSCs for I0, I4, and 21 days after the induction of osteogenic differentiation. Results are mean \pm SE of the triplicate experiments. $* P<0.05$.

Abbreviations: AuNPs, gold nanoparticles; hADMSCs, human adipose-derived mesenchymal stem cells; OM, osteogenic-inducing medium; SE, standard error; OD, optical density.

chitosan enhanced mineralization of bone marrow-derived hMSCs by upregulating genes associated with mineralization and calcium-binding proteins. ${ }^{37,38}$ In particular, multiwall carbon nanotube scaffolds modified with chitosan enhanced proliferation and mineralization of MG-63 cells more than chitosan scaffolds. ${ }^{39}$ In order to confirm the effect of chitosan-conjugated AuNPs on the osteogenic differentiation of hADMSCs in osteogenic-inducing conditions, osteogenic differentiation was determined by measuring the mineralized calcium deposition. The result shows that the mineralized calcium deposition increased more in the hADMSCs containing OM with chitosan-conjugated AuNPs group than in the OM-only group in a time-dependent manner (Figure 3A). In 21 days, the mineralized calcium deposition increased in the containing AuNPs groups up to 1.4 -fold at a concentration of $0.5 \mathrm{ppm}$ and 1.35 -fold at $1 \mathrm{ppm}$, as compared to the OM-only group (Figure 3B). Therefore, chitosan-conjugated AuNPs stimulated the differentiation of hADMSCs by osteogenesis.

\section{Expression of osteogenic differentiation specific genes in hADMSCs}

A real-time RT-PCR analysis was used to measure the expressions of specific genes to reveal the involvement of the osteogenic differentiation of hADMSCs in OM-containing chitosan-conjugated AuNPs. In the osteogenic differentiation of hMSCs, bone formation is a complex biological process involving several tightly regulated gene expression patterns of bone-related proteins, and specific marker genes, such as osteocalcin (OSC), osteopontin, and bone sialoprotein (BSP), were expressed according to the progress of differentiation. ${ }^{40}$ Alkaline phosphatase (ALP) is a key regulator of the early stage differentiation of bone marrow stromal cells, and gradually decreased as proceeding through differentiation into osteoblasts. ${ }^{41}$ The result shows that the ALP mRNA expression level increased more in the cells containing chitosanconjugated AuNPs group as compared to the OM-only group at 10 days (Figure 4A). The ALP mRNA expression level in the OM-only group showed a maximal value at 14 days, and it decreased at 21 days. In the OM-containing chitosanconjugated AuNPs group, the ALP mRNA expression levels increased at 10 days when compared to the OM-only group, and the levels were sustained at 14 days and 21 days. Lian and Stein suggest that the ALP mRNA expression changed during the developmental maturation stage of the osteoblast phenotype. ${ }^{42}$ The peak levels of the ALP mRNA represent the matrix maturation period, and the cellular levels of the ALP mRNA in the heavily mineralized stage decreased. ${ }^{42}$ We observed that the ALP mRNA expression was early induced by the chitosan-conjugated AuNPs group, as compared to the OM-only group. The BSP gene is up-regulated by promoting bone formation. ${ }^{43}$ The BSP mRNA expression levels in the OM-containing chitosan-conjugated AuNPs group at $0.5 \mathrm{ppm}$ and $1 \mathrm{ppm}$ were higher than in the OM-only group at 14 days and 21 days (Figure 4B). In the OM-only group, the BSP mRNA level decreased in a time-dependent manner. However, the BSP mRNA level in the OM-containing chitosanconjugated AuNPs group increased up to 14 days and then decreased at 21 days, as compared to the OM-only group. This indicates the BSP mRNA expression was stimulated 
A
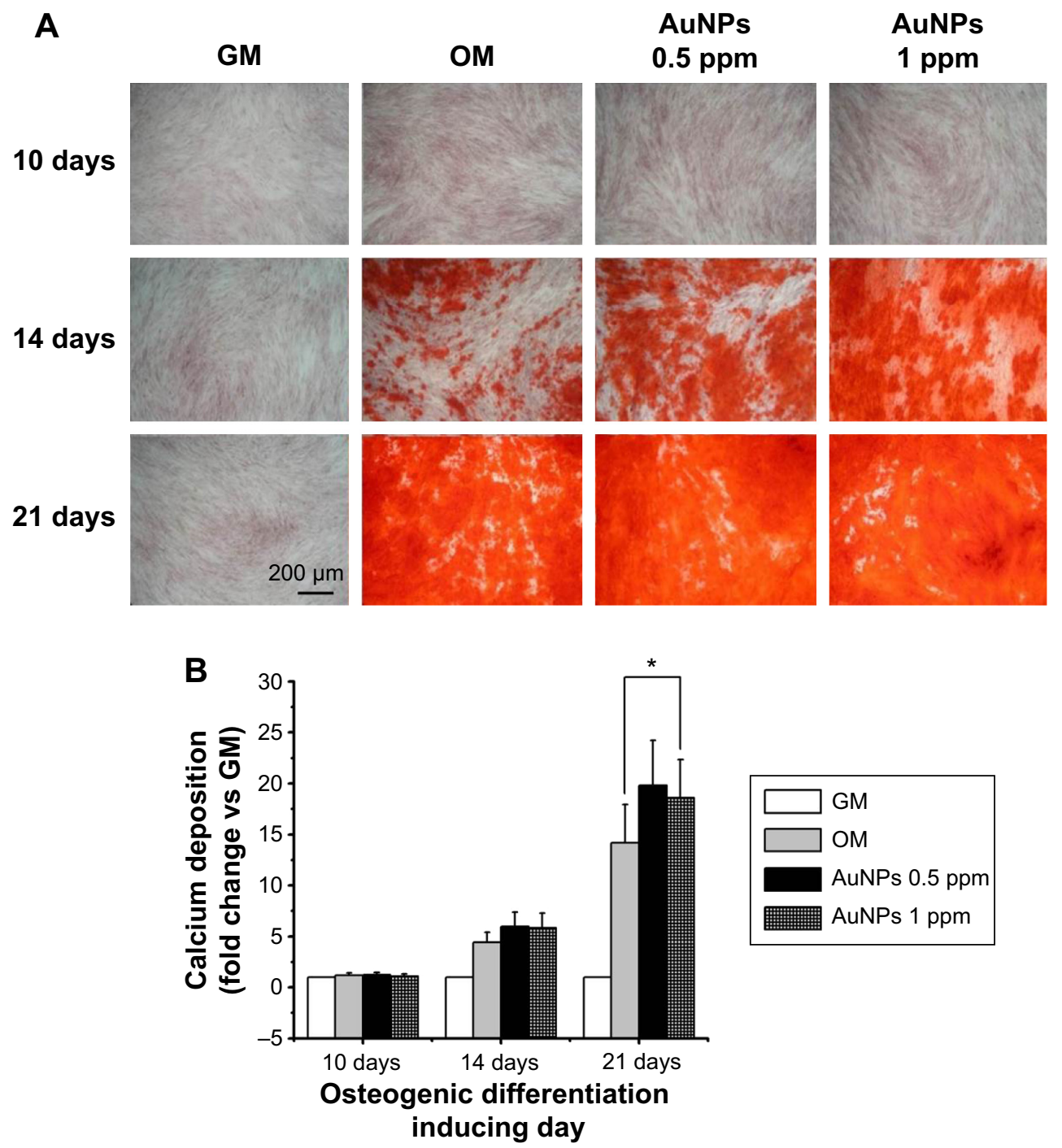

Figure 3 Effect of chitosan-conjugated AuNPs on the mineralization of hADMSCs.

Notes: (A) Stained calcium deposition by Alizarin Red S. (B) Mineralization was quantitated through the elution of Alizarin Red S from stained mineral deposits. Results are mean \pm SE of the triplicate experiments, ${ }^{*} P<0.05$.

Abbreviations: AuNPs, gold nanoparticles; GM, growth medium; hADMSCs, human adipose-derived mesenchymal stem cells; OM, osteogenic-inducing medium; SE, standard error.

by the chitosan-conjugated AuNPs. The OSC gene is a key marker for the late maturation of osteoblasts and the expression of OSC is concomitantly up-regulated by more advanced osteoblasts. ${ }^{40,44}$ The OSC mRNA expression levels increased in cells induced by osteogenic differentiation, and the OSC mRNA expression levels in the OM-containing chitosanconjugated AuNPs group were significantly higher than in the OM-only group at 10 days and 14 days (Figure 4C). Thus, the cell fate of the hADMSCs was more induced by the chitosan-conjugated AuNPs group into osteoblasts than by the OM-only group.

According to the integrated real-time PCR results, the expressions of the osteogenic marker genes were more activated by the chitosan-conjugated AuNPs group, and the osteogenic differentiation of hADMSCs treated with chitosan-conjugated AuNPs was more promoted than in the OM-only group.

\section{Chitosan-conjugated AuNPs do not affect the adipogenic differentiation in osteogenic-inducing hADMSCs}

Osteogenesis and adipogenesis are reciprocal events sharing some transcription factors in the specific lineage fate of MSCs. ${ }^{45}$ The stimulation of osteogenic differentiation by AuNPs inhibits the production of lipid droplets, which is a phenotype marker of the maturation of adipogenic differentiation. ${ }^{22}$ The effect of chitosan-conjugated AuNPs on the adipogenic differentiation of hADMSCs in osteogenicinducing conditions was determined by measuring lipid droplets stained by ORO dye. In our study, the accumulation 

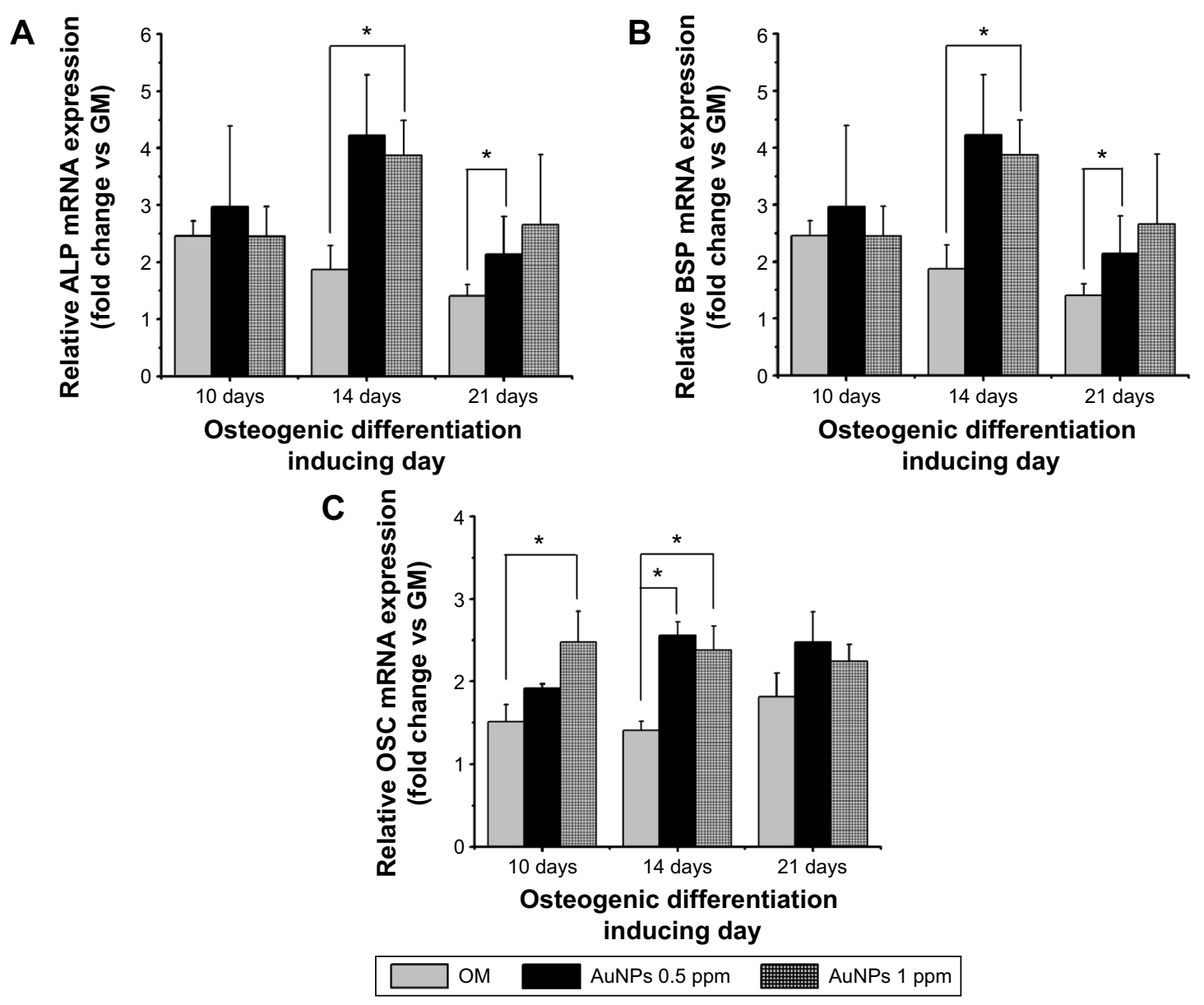

Figure 4 A real-time RT-PCR analysis of the osteogenic marker gene expression in hADMSCs treated with chitosan-conjugated AuNPs for I0, I4, and 2I days. Notes: (A) Alkaline phosphatase (ALP); (B) bone sialoprotein (BSP); (C) osteocalcin (OSC). Results are mean \pm SE of the triplicate experiments. $* P<0.05$. Abbreviations: AuNPs, gold nanoparticles; GM, growth medium; hADMSCs, human adipose-derived mesenchymal stem cells; OM, osteogenic-inducing medium; RT-PCR, reverse transcription polymerase chain reaction; SE, standard error.

of lipid droplets was slightly increased in the OM-only group and the OM-containing chitosan-conjugated AuNPs group (Figure 5). However, the degree of accumulation was weak when compared to the mineralization, and it was similar between the OM-only group and the OM-containing chitosan-conjugated AuNPs group. Therefore, the adipogenic differentiation of hADMSCs was not induced by chitosanconjugated AuNPs in OM conditions.

\section{Chitosan-conjugated AuNPs enhance the osteogenic differentiation of hADMSCs through the $\mathrm{Wnt} / \beta$-catenin signaling pathway}

Several studies demonstrated that the $\mathrm{Wnt} / \beta$-catenin signaling pathway was initially involved in osteoblast differentiation. ${ }^{46-48} \mathrm{Wnt} / \beta$-catenin signaling inhibits adipogenic differentiation and alters the cell fate from adipocyte to osteoblast in hADMSCs. ${ }^{49}$ To confirm the signaling

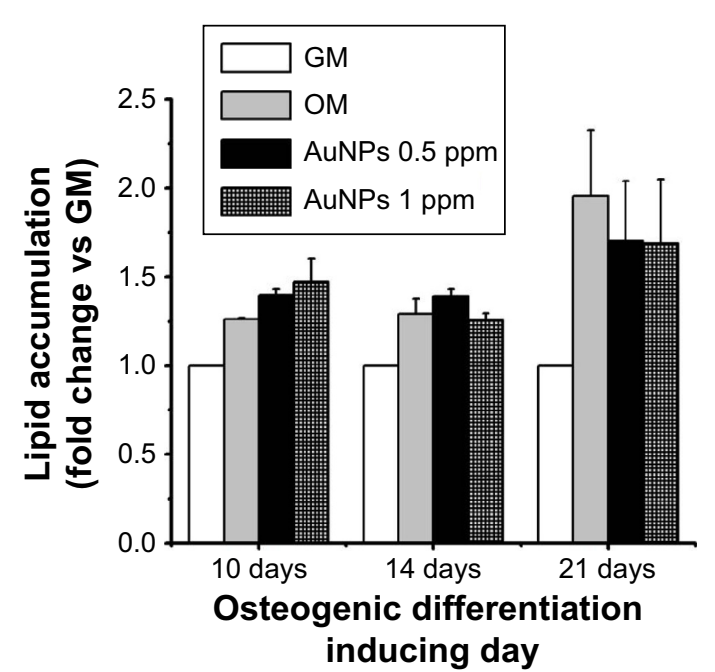

Figure 5 Effect of chitosan-conjugated AuNPs on adipogenic differentiation. Notes: Lipid accumulation of hADMSCs treated with chitosan-conjugated AuNPs after 10, 14, and 21 days of culturing. Results are mean \pm SE of the triplicate experiments.

Abbreviations: AuNPs, gold nanoparticles; GM, growth medium; hADMSCs, human adipose-derived mesenchymal stem cells; OM, osteogenic-inducing medium; SE, standard error. 
pathway related to osteogenic differentiation in hADMSCs, we examined nonphosphorylated $\beta$-catenin in protein levels. Nonphosphorylated $\beta$-catenin protein levels increased in the OM-containing chitosan-conjugated AuNPs group at 7 days, as compared to the OM-only group (Figure 6A). The nonphosphorylated $\beta$-catenin in the cytoplasm accumulated and then translocated into the nucleus while activating the Wnt/ $\beta$-catenin signaling pathway. ${ }^{50-52}$ The translocation of nonphosphorylated $\beta$-catenin into the nuclei of the OM group was determined by using an immunofluorescent antibody (Figure 6B). The translocation of nonphosphorylated $\beta$-catenin into nuclei of the OM-containing chitosanconjugated AuNPs group was greater than in the OM-only group at 7 days and 10 days. After 10 days, the degree of translocated nonphosphorylated $\beta$-catenin proteins was similar between the OM-only group and the OM-containing
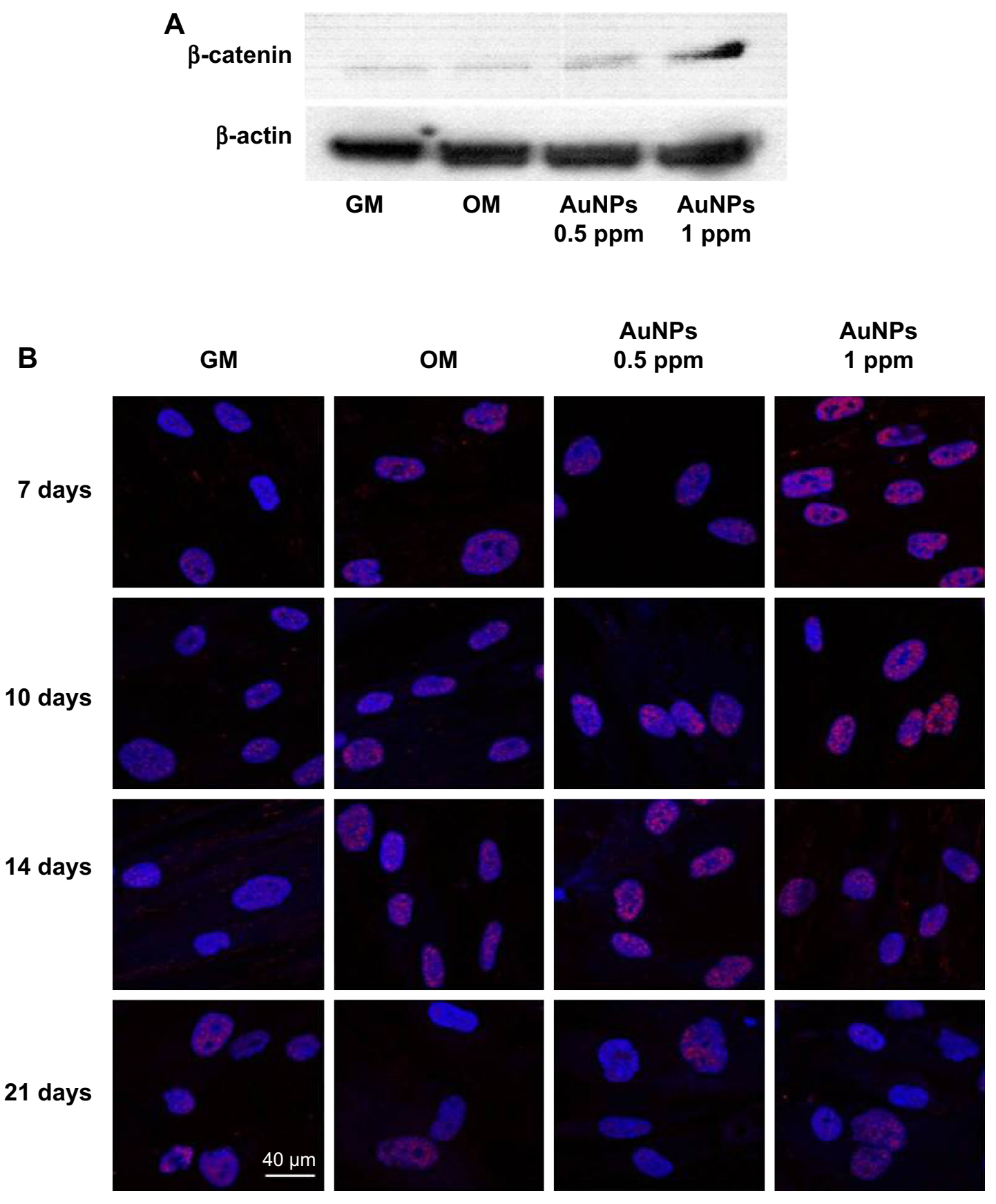

Figure 6 Activation of the osteogenic signaling pathway by chitosan-conjugated AuNPs.

Notes: (A) A western blot analysis of the nonphosphorylated $\beta$-catenin protein expression after treatment with chitosan-conjugated AuNPs for 7 days. (B) Translocation of nonphosphorylated $\beta$-catenin into the nuclei of hADMSCs treated with chitosan-conjugated AuNPs for $7,10,14$, and 21 days.

Abbreviations: AuNPs, gold nanoparticles; GM, growth medium; hADMSCs, human adipose-derived mesenchymal stem cells; OM, osteogenic-inducing medium. 
chitosan-conjugated AuNPs group. Some studies suggested that various type of NPs act in hMSCs by different action mechanism. Adipogenic and osteogenic differentiation of hMSCs added with silver NPs was inhibited by the $\mathrm{Ag}^{+}$ ion, which suppresses the expression of marker genes, and SH-SY5Y cells treated with silver NPs differentiate into neuronal cells through ROS activating the ERK-AKT pathway. ${ }^{53,54}$ Also, AuNPs enhance the osteogenic differentiation of MSCs through the activation of the mitogen-activated protein kinase and bone morphogenic protein signaling pathways. ${ }^{22,23}$ However, the mechanism of the action of the NPs in hADMSCs has not been clear. AuNPs induced mechanical stimulation in MSCs. ${ }^{22}$ Also, several researches suggested that mechanical stimulation enhance osteogenesis and inhibit adipogenesis through activation of $\mathrm{Wnt} / \beta$-catenin signaling. ${ }^{55-58}$

According to our results, mechanical stimulation by uptake of chitosan-conjugated AuNPs in hADMSCs promote the differentiation of hADMSCs into osteoblasts through nonphosphorylated $\beta$-catenin accumulation, as it enhances the switching of the hADMSC cell fate from adipocyte to osteoblast.

\section{Conclusion}

This study showed that chitosan-conjugated AuNPs at nontoxic concentrations enhanced the osteogenic differentiation of hADMSCs. The uptake of chitosan-conjugated AuNPs in hADMSCs induced mechanical stimulation that regulated mRNA expression and mineralization by activating the $\mathrm{Wnt} / \beta$-catenin signaling pathway. Therefore, chitosan-conjugated AuNPs have been demonstrated as stimulators of the osteogenic differentiation of MSCs, and can be used as vehicles for promoting bone tissue regeneration.

\section{Acknowledgment}

This research was supported by Basic Science Research Program through National Research Foundation of Korea (NRF) funded by the Ministry of Science, ICT \& Future Planning (NRF-2014R1A1A3A04052757).

\section{Disclosure}

The authors report no conflicts of interest in this work.

\section{References}

1. Oyen J, Brudvik C, Gjesdal CG, Tell GS, Lie SA, Hove LM. Osteoporosis as a risk factor for distal radial fractures: a case-control study. $J$ Bone Joint Surg Am. 2011;93(4):348-356.

2. McDonald JW, Liu XZ, Qu Y, et al. Transplanted embryonic stem cells survive, differentiate and promote recovery in injured rat spinal cord. Nat Med. 1999;5(12):1410-1412.
3. Ren G, Chen X, Dong F, et al. Concise review: mesenchymal stem cells and translational medicine: emerging issues. Stem Cells Transl Med. 2012;1(1):51-58.

4. Maxson S, Lopez EA, Yoo D, Danilkovitch-Miagkova A, Leroux MA Concise review: role of mesenchymal stem cells in wound repair. Stem Cells Transl Med. 2012;1(2):142-149.

5. Sanfelix-Genoves J, Hurtado I, Sanfelix-Gimeno G, Reig-Molla B, Peiro S. Impact of osteoporosis and vertebral fractures on quality-oflife. A population-based study in Valencia, Spain (The FRAVO Study). Health Qual Life Outcomes. 2011;9:20.

6. Compston JE, Flahive J, Hooven FH, et al. Obesity, health-care utilization, and health-related quality of life after fracture in postmenopausal women: Global Longitudinal Study of Osteoporosis in Women (GLOW). Calcif Tissue Int. 2014;94(2):223-231.

7. Pittenger MF, Mackay AM, Beck SC, et al. Multilineage potential of adult human mesenchymal stem cells. Science. 1999;284(5411):143-147.

8. Gimble J, Guilak F. Adipose-derived adult stem cells: isolation, characterization, and differentiation potential. Cytotherapy. 2003;5(5):362-369.

9. Wagner W, Wein F, Seckinger A, et al. Comparative characteristics of mesenchymal stem cells from human bone marrow, adipose tissue, and umbilical cord blood. Exp Hematol. 2005;33(11):1402-1416.

10. Kern S, Eichler H, Stoeve J, Kluter H, Bieback K. Comparative analysis of mesenchymal stem cells from bone marrow, umbilical cord blood, or adipose tissue. Stem Cells. 2006;24(5):1294-1301.

11. Toupadakis CA, Wong A, Genetos DC, et al. Comparison of the osteogenic potential of equine mesenchymal stem cells from bone marrow, adipose tissue, umbilical cord blood, and umbilical cord tissue. Am $J$ Vet Res. 2010;71(10):1237-1245.

12. Chen HT, Lee MJ, Chen CH, et al. Proliferation and differentiation potential of human adipose-derived mesenchymal stem cells isolated from elderly patients with osteoporotic fractures. J Cell Mol Med. 2012;16(3): $582-593$.

13. Vemuri MC, Chase LG, Rao MS. Mesenchymal stem cell assays and applications. Methods Mol Biol. 2011;698:3-8.

14. Peer D, Karp JM, Hong S, Farokhzad OC, Margalit R, Langer R. Nanocarriers as an emerging platform for cancer therapy. Nat Nanotechnol. 2007;2(12):751-760.

15. Zhang L, Gu FX, Chan JM, Wang AZ, Langer RS, Farokhzad OC. Nanoparticles in medicine: therapeutic applications and developments. Clin Pharmacol Ther. 2008;83(5):761-769.

16. Wang M, Li Z, Qiao H, Chen L, Fan Y. Effect of gold/Fe3O4 nanoparticles on biocompatibility and neural differentiation of rat olfactory bulb neural stem cells. J Nanomater. 2013;2013:867426.

17. Kim TH, Lee KB, Choi JW. 3D graphene oxide-encapsulated gold nanoparticles to detect neural stem cell differentiation. Biomaterials. 2013; 34(34):8660-8670.

18. Orza A, Soritau O, Olenic L, et al. Electrically conductive gold-coated collagen nanofibers for placental-derived mesenchymal stem cells enhanced differentiation and proliferation. ACS Nano. 2011;5(6): 4490-4503.

19. Ravichandran R, Sridhar R, Venugopal JR, Sundarrajan S, Mukherjee S, Ramakrishna S. Gold nanoparticle loaded hybrid nanofibers for cardiogenic differentiation of stem cells for infarcted myocardium regeneration. Macromol Biosci. 2014;14(4):515-525.

20. Phillips JE, Petrie TA, Creighton FP, Garcia AJ. Human mesenchymal stem cell differentiation on self-assembled monolayers presenting different surface chemistries. Acta Biomater. 2010;6(1):12-20.

21. Zhang D, Liu D, Zhang J, Fong C, Yang M. Gold nanoparticles stimulate differentiation and mineralization of primary osteoblasts through the ERK/MAPK signaling pathway. Mater Sci Eng C Mater Biol Appl. 2014; 42:70-77.

22. Yi C, Liu D, Fong CC, Zhang J, Yang M. Gold nanoparticles promote osteogenic differentiation of mesenchymal stem cells through p38 MAPK pathway. ACS Nano. 2010;4(11):6439-6448.

23. Heo DN, Ko WK, Bae MS, et al. Enhanced bone regeneration with a gold nanoparticle-hydrogel complex. JMater Chem B Mater Biol Med. 2014(2):1584-1594. 
24. Ilie I, Ilie R, Mocan T, Bartos D, Mocan L. Influence of nanomaterials on stem cell differentiation: designing an appropriate nanobiointerface. Int J Nanomedicine. 2012;7:2211-2225.

25. Choi SY, Jang SH, Park J, et al. Cellular uptake and cytotoxicity of positively charged chitosan gold nanoparticles in human lung adenocarcinoma cells. J Nanopart Res. 2012;14(12).

26. Cho EC, Xie J, Wurm PA, Xia Y. Understanding the role of surface charges in cellular adsorption versus internalization by selectively removing gold nanoparticles on the cell surface with a I2/KI etchant. Nano Lett. 2009;9(3):1080-1084.

27. Lin J, Zhang H, Chen Z, Zheng Y. Penetration of lipid membranes by gold nanoparticles: insights into cellular uptake, cytotoxicity, and their relationship. ACS Nano. 2010;4(9):5421-5429.

28. Verma A, Uzun O, Hu Y, et al. Surface-structure-regulated cell-membrane penetration by monolayer-protected nanoparticles. Nat Mater. 2008; 7(7):588-595.

29. Kim JA, Aberg C, Salvati A, Dawson KA. Role of cell cycle on the cellular uptake and dilution of nanoparticles in a cell population. Nat Nanotechnol. 2012;7(1):62-68.

30. Kuhn DA, Vanhecke D, Michen B, et al. Different endocytotic uptake mechanisms for nanoparticles in epithelial cells and macrophages. Beilstein J Nanotechnol. 2014;5:1625-1636.

31. Fan JH, Li WT, Hung WI, Chen CP, Yeh JM. Cytotoxicity and differentiation effects of gold nanoparticles to human bone marrow mesenchymal stem cells. Biomed Eng Appl Basis Commun. 2011;23(2):141-152.

32. Atashi F, Modarressi A, Pepper MS. The role of reactive oxygen species in mesenchymal stem cell adipogenic and osteogenic differentiation: a review. Stem Cells Dev. 2015; 24(10):1150-1163.

33. Lennon DP, Haynesworth SE, Bruder SP, Jaiswal N, Caplan AI. Human and animal mesenchymal progenitor cells from bone marrow: identification of serum for optimal selection and proliferation. In Vitro Cell Dev Biol Anim. 1996;32:602-611.

34. Xia T, Kovochich M, Brant J, et al. Comparison of the abilities of ambient and manufactured nanoparticles to induce cellular toxicity according to an oxidative stress paradigm. Nano Lett. 2006;6(8):1794-1807.

35. Dam DH, Lee JH, Sisco PN, et al. Direct observation of nanoparticlecancer cell nucleus interactions. ACS Nano. 2012;6(4):3318-3326.

36. Alanazi FK, Radwan AA, Alsarra IA. Biopharmaceutical applications of nanogold. Saudi Pharm J. 2010;18(4):179-193.

37. Muzzarelli RAA. Chitosan composites with inorganics, morphogenetic proteins and stem cells, for bone regeneration. Carbohydr Polym. 2011; 83(4):1433-1445.

38. Mathews S, Gupta PK, Bhonde R, Totey S. Chitosan enhances mineralization during osteoblast differentiation of human bone marrow-derived mesenchymal stem cells, by upregulating the associated genes. Cell Prolif. 2011;44(6):537-549.

39. Venkatesan J, Ryu B, Sudha PN, Kim SK. Preparation and characterization of chitosan-carbon nanotube scaffolds for bone tissue engineering. Int J Biol Macromol. 2012;50(2):393-402.

40. Stein GS, Lian JB. Molecular mechanisms mediating proliferation/ differentiation interrelationships during progressive development of the osteoblast phenotype. Endocr Rev. 1993;14(4):424-442.

41. Malaval L, Modrowski D, Gupta AK, Aubin JE. Cellular expression of bone-related proteins during in vitro osteogenesis in rat bone marrow stromal cell cultures. J Cell Physiol. 1994;158(3):555-572.
42. Lian JB, Stein GS. Development of the osteoblast phenotype: molecular mechanisms mediating osteoblast growth and differentiation. Iowa Orthop J. 1995;15:118-140.

43. Ganss B, Kim RH, Sodek J. Bone sialoprotein. Crit Rev Oral Biol Med. 1999;10(1):79-98.

44. Choi MH, Noh WC, Park JW, Lee JM, Suh JY. Gene expression pattern during osteogenic differentiation of human periodontal ligament cells in vitro. J Periodontal Implant Sci. 2011;41(4):167-175.

45. Takada I, Kouzmenko AP, Kato S. Molecular switching of osteoblastogenesis versus adipogenesis: implications for targeted therapies. Expert Opin Ther Targets. 2009;13(5):593-603.

46. Heo JS, Lee SY, Lee JC. Wnt/beta-catenin signaling enhances osteoblastogenic differentiation from human periodontal ligament fibroblasts. Mol Cells. 2010;30(5):449-454.

47. Guidotti S, Facchini A, Platano D, et al. Enhanced osteoblastogenesis of adipose-derived stem cells on spermine delivery via beta-catenin activation. Stem Cells Dev. 2013;22(10):1588-1601.

48. D'Alimonte I, Lannutti A, Pipino C, et al. Wnt signaling behaves as a "master regulator" in the osteogenic and adipogenic commitment of human amniotic fluid mesenchymal stem cells. Stem Cells Rev. 2013;9(5): $642-654$.

49. Li HX, Luo X, Liu RX, Yang YJ, Yang GS. Roles of Wnt/beta-catenin signaling in adipogenic differentiation potential of adipose-derived mesenchymal stem cells. Mol Cell Endocrinol. 2008;291(1-2):116-124.

50. Clevers H. Wnt/beta-catenin signaling in development and disease. Cell. 2006;127(3):469-480.

51. Takahashi-Yanaga F, Sasaguri T. The Wnt/beta-catenin signaling pathway as a target in drug discovery. J Pharmacol Sci. 2007;104(4): 293-302.

52. Olivares-Navarrete R, Hyzy SL, Hutton DL, et al. Role of non-canonical Wnt signaling in osteoblast maturation on microstructured titanium surfaces. Acta Biomater. 2011;7(6):2740-2750.

53. Sengstock C, Diendorf J, Epple M, Schildhauer TA, Koller M. Effect of silver nanoparticles on human mesenchymal stem cell differentiation. Beilstein J Nanotechnol. 2014;5:2058-2069.

54. Dayem AA, Kim B, Gurunathan S, et al. Biologically synthesized silver nanoparticles induce neuronal differentiation of SH-SY5Y cells via modulation of reactive oxygen species, phosphatases, and kinase signaling pathways. Biotechnol J. 2014;9(7):934-943.

55. Robinson JA, Chatterjee-Kishore M, Yaworsky PJ, et al. Wnt/betacatenin signaling is a normal physiological response to mechanical loading in bone. J Biol Chem. 2006;281(42):31720-31728.

56. Case N, Ma M, Sen B, Xie Z, Gross TS, Rubin J. Beta-catenin levels influence rapid mechanical responses in osteoblasts. J Biol Chem. 2008; 283(43):29196-29205.

57. Sen B, Xie Z, Case N, Ma M, Rubin C, Rubin J. Mechanical strain inhibits adipogenesis in mesenchymal stem cells by stimulating a durable beta-catenin signal. Endocrinology. 2008;149(12):6065-6075.

58. Niziolek PJ, Warman ML, Robling AG. Mechanotransduction in bone tissue: the A214V and G171V mutations in Lrp5 enhance loadinduced osteogenesis in a surface-selective manner. Bone. 2012;51(3): 459-465.
International Journal of Nanomedicine

\section{Publish your work in this journal}

The International Journal of Nanomedicine is an international, peerreviewed journal focusing on the application of nanotechnology in diagnostics, therapeutics, and drug delivery systems throughout the biomedical field. This journal is indexed on PubMed Central, MedLine, CAS, SciSearch ${ }^{\circledR}$, Current Contents ${ }^{\circledR} /$ Clinical Medicine,

\section{Dovepress}

Journal Citation Reports/Science Edition, EMBase, Scopus and the Elsevier Bibliographic databases. The manuscript management system is completely online and includes a very quick and fair peer-review system, which is all easy to use. Visit http://www.dovepress.com/ testimonials.php to read real quotes from published authors. 